\title{
Near-Infrared Light Activation of Proteins Inside Living Cells Enabled by Carbon Nanotube- Mediated Intracellular Delivery
}

\author{
He Li, Xinqi Fan, and Xing Chen*
}

Beijing National Laboratory for Molecular Sciences, Key Laboratory of Bioorganic Chemistry and Molecular Engineering of Ministry of Education, College of Chemistry and Molecular Engineering, Synthetic and Functional Biomolecules Center, Peking-Tsinghua Center for Life Sciences, Peking University, Beijing 100871, China.

Corresponding Author Email: xingchen@pku.edu.cn 
Table of contents

Figure S1.

Figure S2.

Figure S3.

Figure S4.

Figure S5.

Figure S6.

Figure S7.

Figure S8.

Figure S9.

Experimental Procedure 

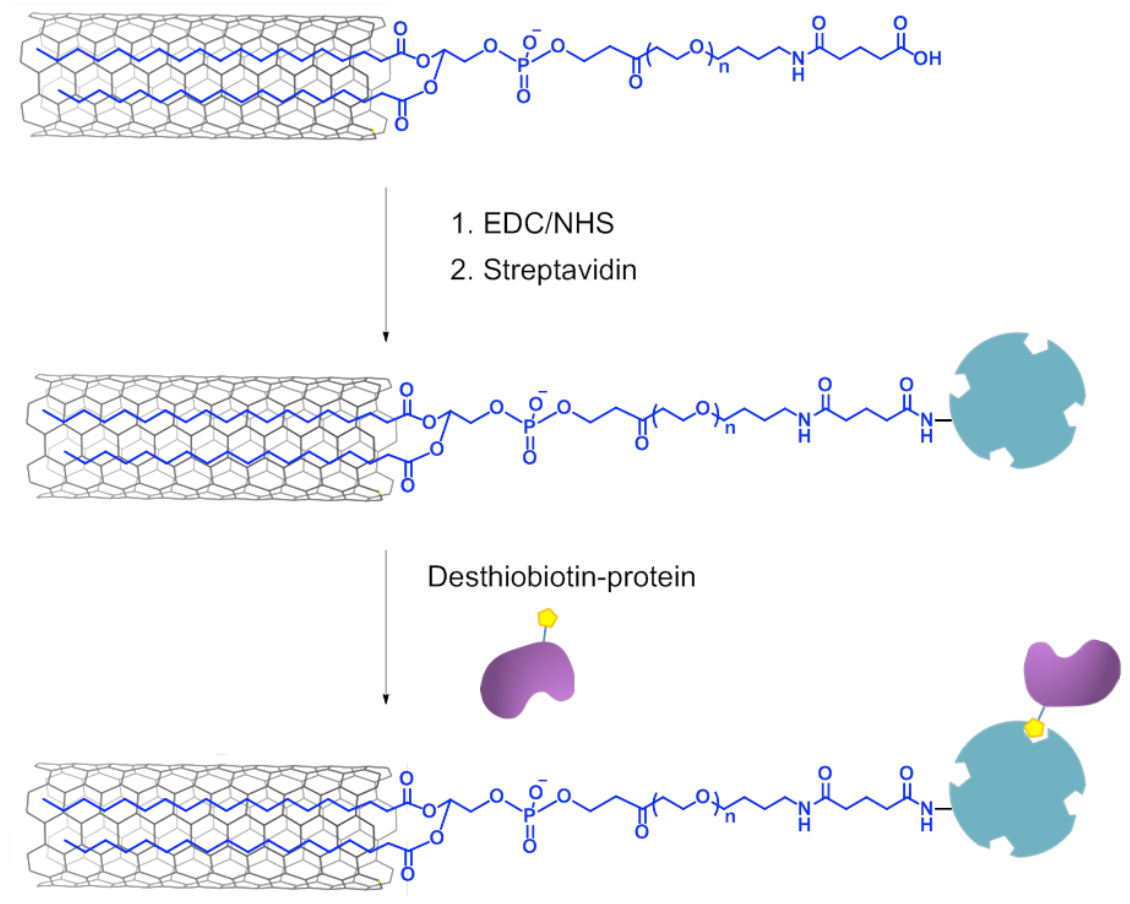

Figure S1. Schematic of the synthesis of SWCNT-protein conjugates.

(a)

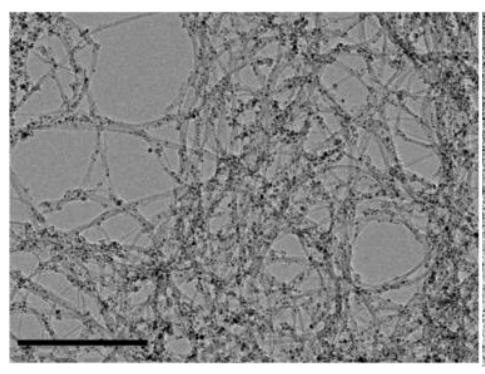

(b)

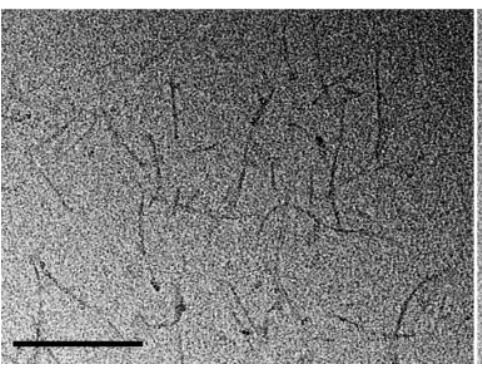

(c)

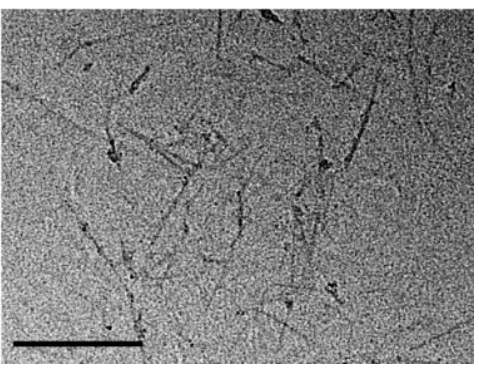

Figure S2. Transmission electron microscopy (TEM) images of SWCNTs and the conjugates. (a) SWCNTs. (b) SWCNT-DSPE-PEG ${ }_{2000}-\mathrm{COOH}$. (c) SWCNT-SA-DTB-EGFP. Scale bars: $200 \mathrm{~nm}$. 


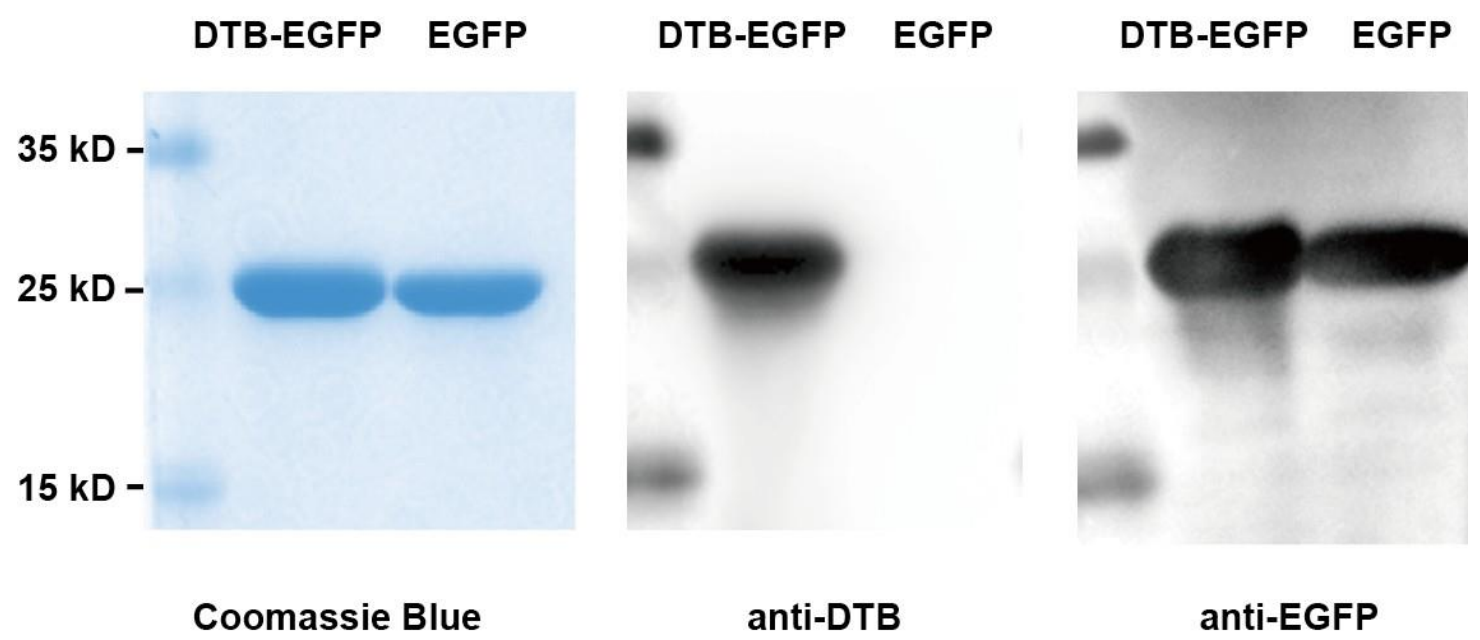

Figure S3. SDS-PAGE and Western blot analysis of DTB-EGFP. The prepared DTB-EGFP was resolved by SDS-PAGE (left) and immunoblotting against DTB (middle) and EGFP (right) using SA-HRP and EGFP antibody respectively. EGFP was shown for comparison. Only DTB-EGFP was visualized on the anti-DTB blot, indicating the chemical conjugation of DTB onto EGFP.

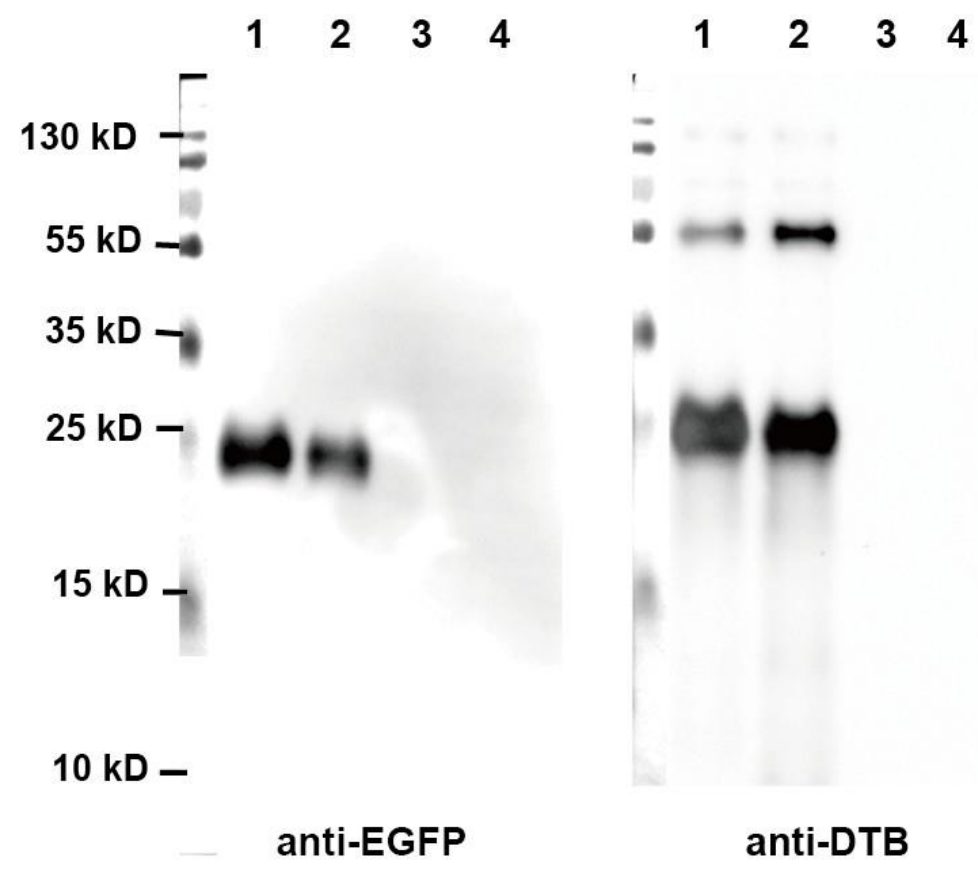

Figure S4. Western Blot analysis of SWCNT-protein conjugates. Lane 1: DTB-EGFP; Lane 2: SWCNT-SA-DTBEGFP; Lane 3: SWCNT-SA; Lane 4: SA. 


\section{$0.67 \mathrm{~W} / \mathrm{cm}^{2}$}

(a)

$0 \mathrm{~min} 1 \mathrm{~min} 2 \mathrm{~min} 5 \mathrm{~min} 10 \mathrm{~min} 20 \mathrm{~min}$

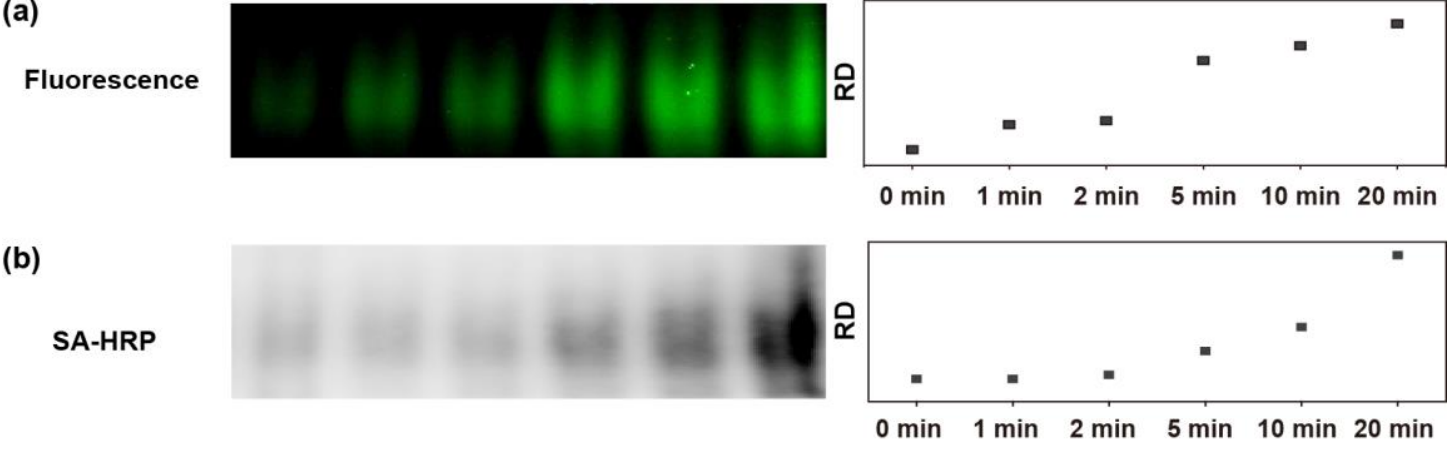

Figure S5. In vitro NIR-triggered release of EGFP at the power density of $0.67 \mathrm{~W} / \mathrm{cm}^{2}$. The solutions of SWCNT-SA-DTB-EGFP were irradiated at $0.67 \mathrm{~W} / \mathrm{cm}^{2}$ for varying time, followed by native PAGE and in-gel fluorescence analysis (a). After in-gel fluorescence scanning, the gel was subjected to Western blot analysis using HRP-conjugated SA (b). Data were quantified by relative density (RD) of the bands.

\section{$1.33 \mathrm{~W} / \mathrm{cm}^{2}$}

(a)
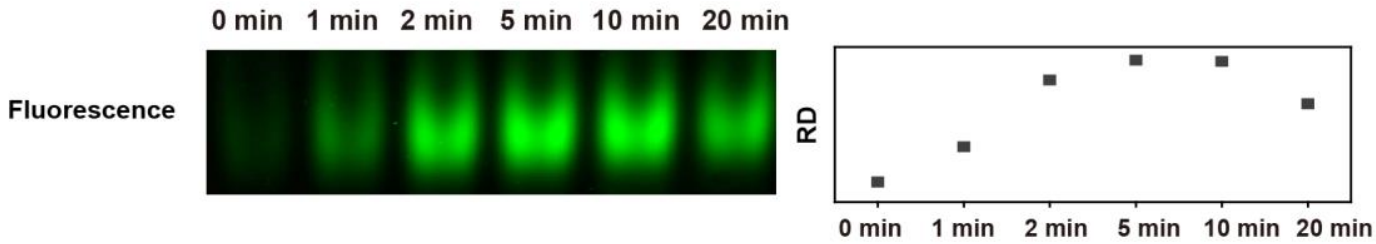

(b)

SA-HRP
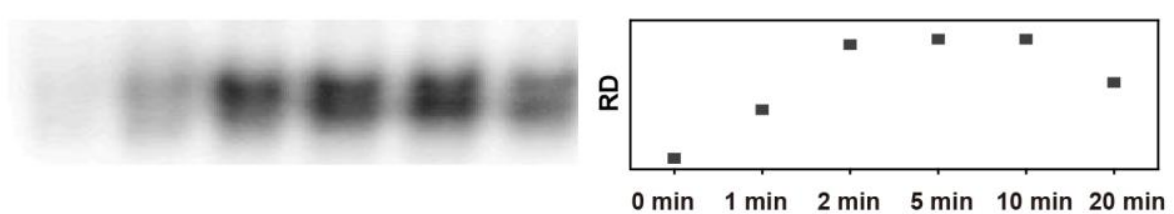

Figure S6. In vitro NIR-triggered release of EGFP at the power density of $1.33 \mathrm{~W} / \mathrm{cm}^{2}$. The solutions of SWCNT-SA-DTB-EGFP were irradiated at $1.33 \mathrm{~W} / \mathrm{cm}^{2}$ for varying time, followed by native PAGE and in-gel fluorescence analysis (a). After in-gel fluorescence scanning, the gel was subjected to Western blot analysis using HRP-conjugated SA (b). Data were quantified by relative density (RD) of the bands. 
(a)

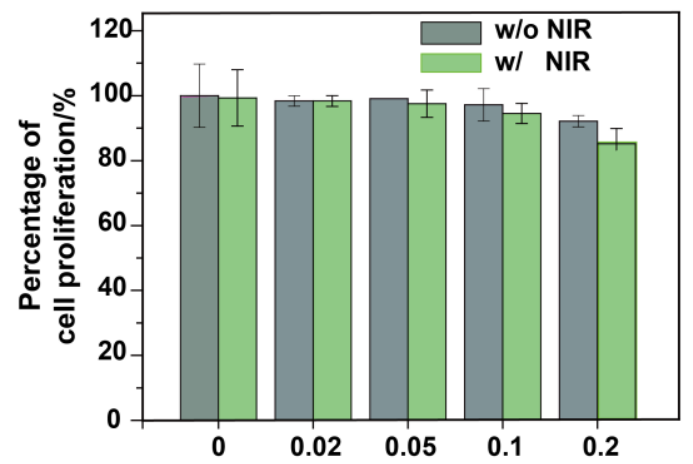

(c) Concentration of SWCNT-SA-DTB-GFP/ $\mu \mathrm{M}$

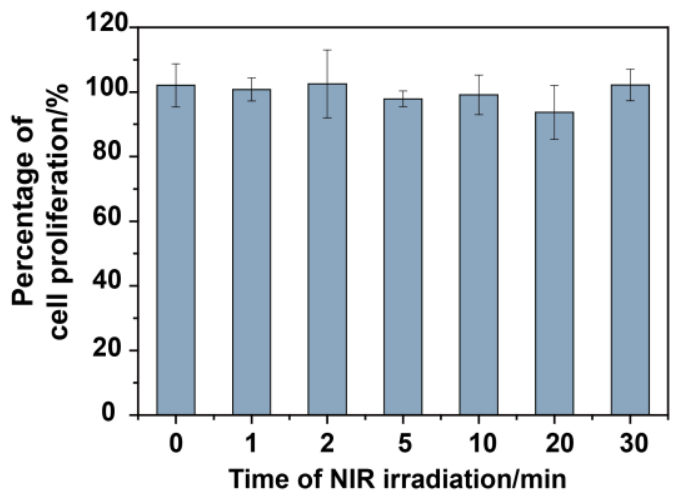

(b)
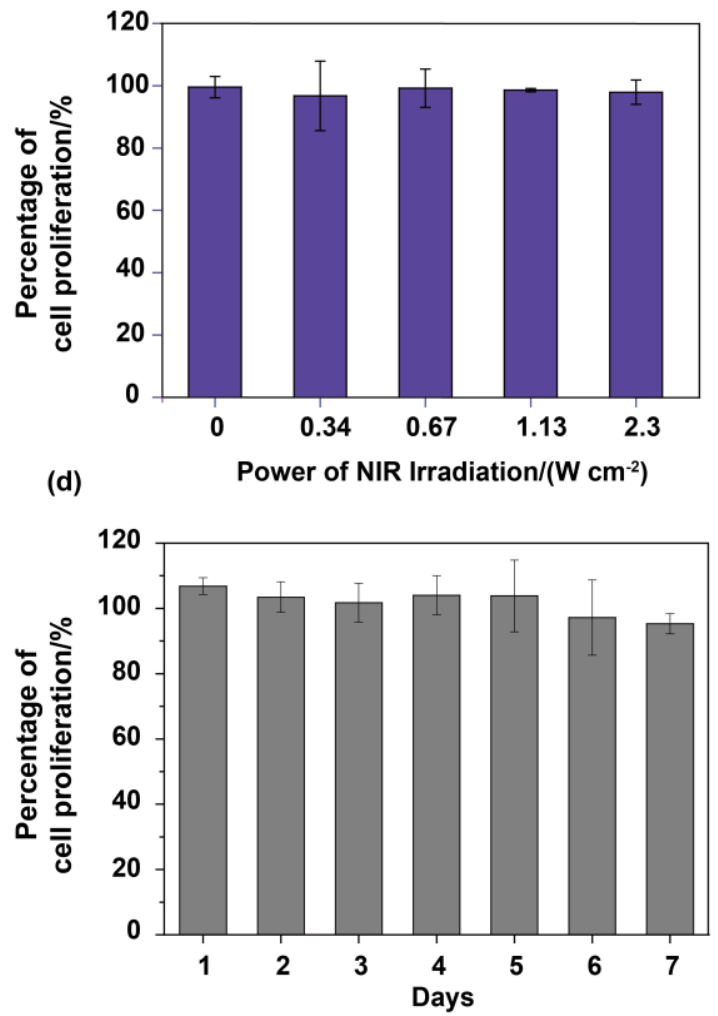

Figure S7. Cell viability analysis by the MTS assay. (a) HeLa cells were incubated with SWCNT-SA-DTB-EGFP at varying concentrations for $6 \mathrm{~h}$, followed by NIR irradiated using the $808 \mathrm{~nm}$ laser (laser power density, 1 $\mathrm{W} / \mathrm{cm}^{2}$; irradiation time, $10 \mathrm{~min}$ ). Cell viability was measured $24 \mathrm{~h}$ later using the MTS assay. (b) Varying power density (concentration of SWCNT-SA-DTB-EGFP, $0.2 \mu \mathrm{M}$; irradiation time, $10 \mathrm{~min}$ ). (c) Varying irradiation time (laser power density, $1.33 \mathrm{~W} / \mathrm{cm}^{2}$; concentration of SWCNT-SA-DTB-EGFP, $0.2 \mu \mathrm{M}$ ). (d) The cells were treated with $0.2 \mu \mathrm{M}$ SWCNT-SA-DTB-EGFP for $6 \mathrm{~h}$, irradiated by $808 \mathrm{~nm}$ laser with power density of $1.33 \mathrm{~W} / \mathrm{cm}^{2}$ for $5 \mathrm{~min}$, and then reseeded into 96 -well plate. The MTS assay was performed on each day for up to 7 days. Error bars represent the standard deviation from six replicate experiments. 


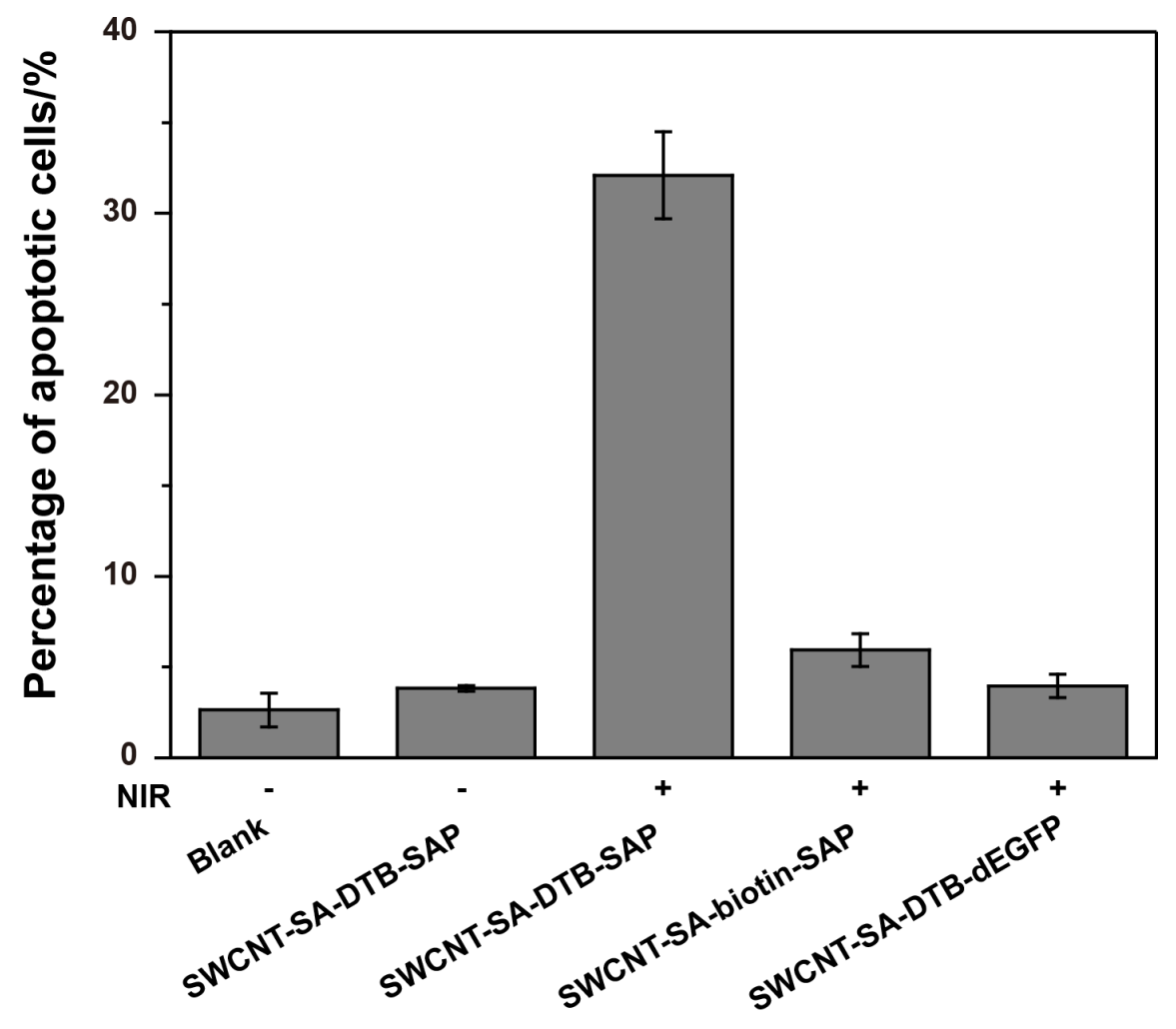

Figure S8. NIR-activated apoptosis in HeLa cells delivered with SWCNT-SA-DTB-SAP. Cells undergoing apoptosis were stained with Annexin $\mathrm{V}$ and $\mathrm{PI}$ and analyzed by flow cytometry. Error bars represent standard deviations from three replicate experiments.

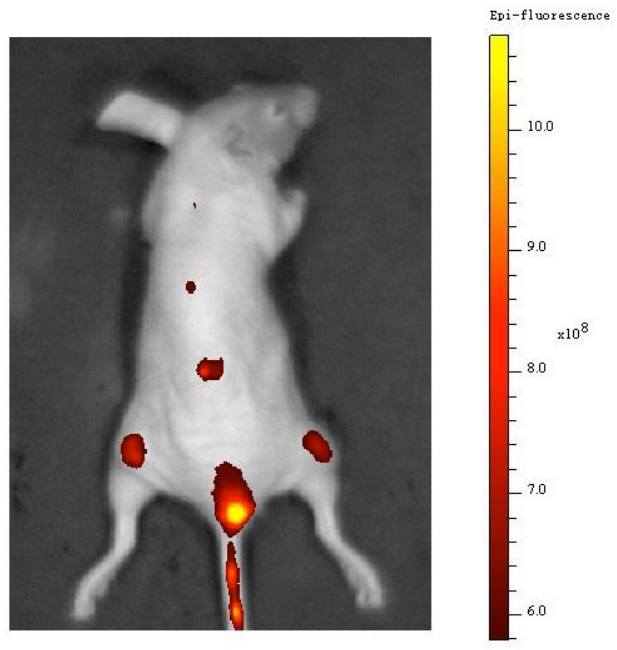

Figure S9. Fluorescence image of tumor-bearing mice after injection of SWCNT-SA-DTB-nEGFP. The mice bearing HeLa cervical cancer xenografts on both flanks were injected with SWCNT-SA-DTB-nEGFP (nEGFP was labelled with Cy5). $4 \mathrm{~h}$ after the injection, the mice were imaged by an IVIS imaging system using the Cy5 channel. 


\section{EXPERIMENTAL PROCEDURES}

Transmission electron microscopy (TEM) characterization of SWCNTs, SWCNT-DSPE-PEG2000-COOH, and SWCNT-SA-DTB-EGFP. $200 \mu \mathrm{l}$ aqueous solution of the SWCNTs, SWCNT-DSPE-PEG $2000-\mathrm{COOH}$, or SWCNTSA-DTB-EGFP suspension was dropped on top of a TEM grid several times, drying in the air and imaged by a Tecnai T20 (FEI, USA) transmission electron microscope.

SDS PAGE and immunoblotting characterization of DTB-EGFP. EGFP and DTB-EGFP were resolved by $12 \%$ SDS-PAGE and immunoblotting using HRP labeled Streptavidin (Beyotime, cat. no. A0303) and antiEGFP rabbit antibody (Cell Signaling, cat. no.2555).

NIR-triggered release of EGFP in vitro. A solution of $0.2 \mu \mathrm{M}$ SWCNT-SA-DTB-EGFP was irradiated by an 808 $\mathrm{nm}$ laser (LOS-BLD-0808-003w-C, Hi-Tech Optoelectronics Co., LTD.) using the designated power density and irradiation time. The solution was then centrifuged at $11,800 \mathrm{~g}$ for $1 \mathrm{~min}$ using a $0.1 \mu \mathrm{m}$ ultrafilter (Millipore, cat. no. UFC30VV). The filtrate was resolved by native PAGE and the gel was directly scanned using a Typhoon fluorescence scanner (GE healthcare, FLA9500). The proteins resolved by the gel were then transferred onto PVDF membrane and detected by streptavidin-HRP (Beyotime, cat. no. A0303).

MTS cell viability assy. HeLa cells were seeded at a density of $1 \times 10^{4}$ cells per well in a 96-well plate containing $100 \mu \mathrm{L}$ medium and cultured for $24 \mathrm{~h}$ before treating with SWCNT-SA-DTB-EGFP at varying concentrations ( 0 to $0.8 \mu \mathrm{M})$. After $6 \mathrm{~h}$, the cells were washed to remove the excess SWCNT-SA-DTBEGFP and irradiated by the $808 \mathrm{~nm}$ laser (power density, 0-5 W/cm ${ }^{2}$ ) for $10 \mathrm{~min}$, or $1 \mathrm{~W} / \mathrm{cm}^{2}$ for varying time. After incubating for another $24 \mathrm{~h}$, the cell viability was assayed using the MTS (3-(4, 5dimethylthiazol-2-yl)-5-(3-carboxymethoxyphenyl)-2-(4-sulfophenyl)-2H-tetrazolium, inner salt) assay (Promega, cat. no. G3582) on a microplate reader (Bio-Tek, Synergy H4). Briefly, MTS (10 $\mu$ L) was added to each well and incubated for $1 \mathrm{~h}$ at $37^{\circ} \mathrm{C}$. The absorbance at $490 \mathrm{~nm}$ was measured for each well. The background absorbance of MTS in cell culture medium was measured. The absorbance of HeLa cells treated with or without SWCNT-SA-DTB-EGFP was separately measured in the absence of MTS. After subtracting the background, the absorbance in each well was normalized to that of control cells with no SWCNT-SA-DTB-EGFP or NIR irradiation. The experiments were carried out in triplicate.

To evaluate the long-term effects of SWCNT-SA-DTB-EGFP on cell growth, HeLa cells were seeded in a 12-well plate and cultured for $24 \mathrm{~h}$ before treating with $0.2 \mu \mathrm{M}$ SWCNT-SA-DTB-EGFP. After $6 \mathrm{~h}$, the cells were washed to remove the excess SWCNT-SA-DTB-EGFP and irradiated by the $808 \mathrm{~nm}$ laser (power density $1.33 \mathrm{~W} / \mathrm{cm}^{2}$ ) for $5 \mathrm{~min}$. The cells were then detached and seeded into a 96-well plate. Cell viability was assayed using the MTS assay on each day for up to 7 days. 